\title{
NOTE ON TRANSLITERATION AND TRANSLATION
}

I have followed the Library of Congress system for transliteration of Russian. Soft signs have been omitted from personal names (Solovev rather than Solov'ev), and I have followed English convention in the case of names with established English spellings (Chernyshevsky, Gorky, Mandelstam, etc.). Unless noted, translations are my own. Where I use a preexisting translation, I have noted any alterations, often made to restore the original text's precision in agronomic or soil terminology. 
